\title{
Callidad percibida de los cuidados de enfermería por pacientes en relación con servicio de hospitalización
}

\author{
Olivetto Ocampo Anel Adriana*, Becerra Murillo Marisol*, Silva Tiscareño Leonardo*, Lozoyo \\ Hernández Mauricio*, González Flores Silvia Patricia**
}

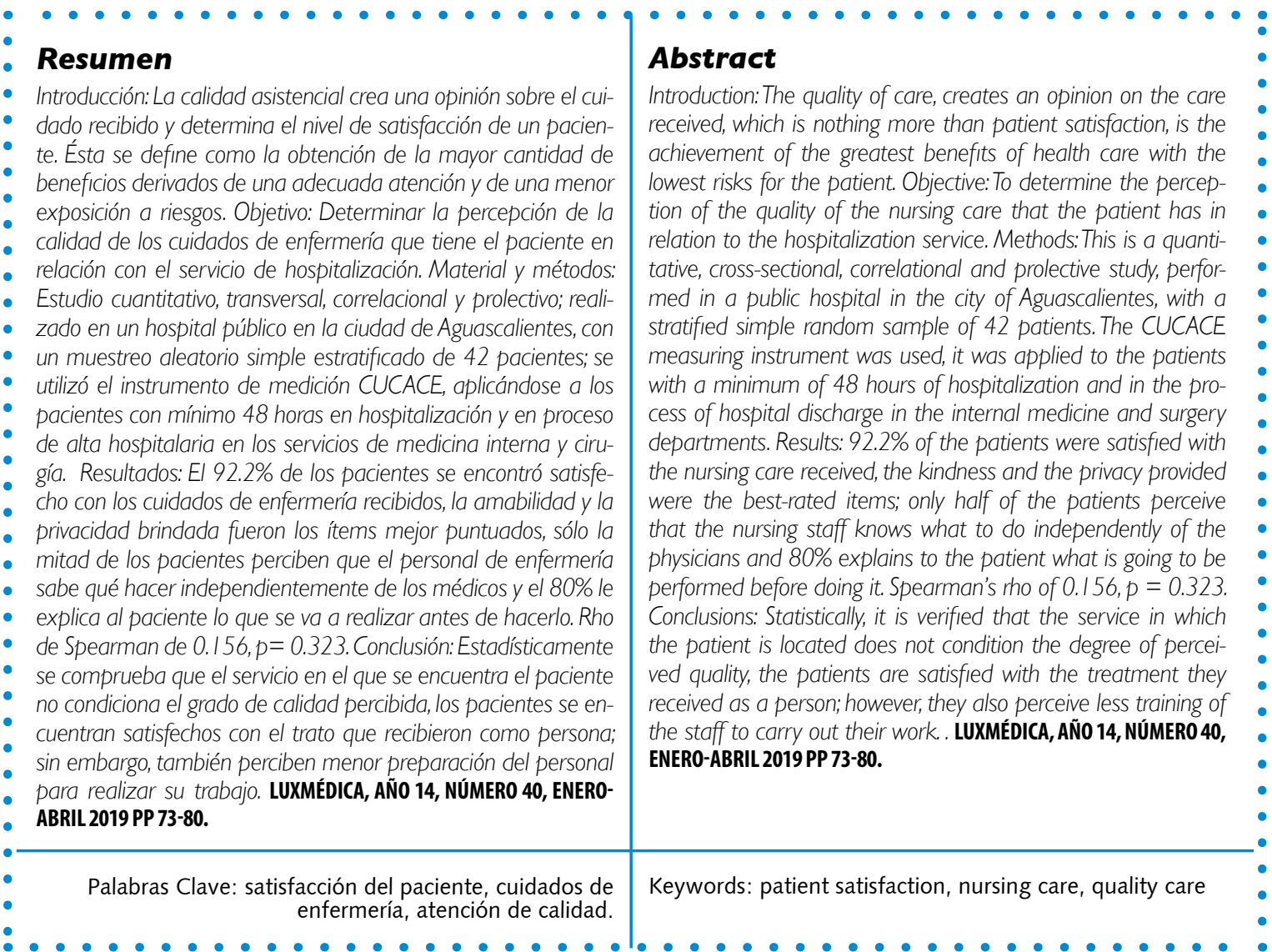

\footnotetext{
Estudiante del octavo semestre de la Licenciatura de Enfermería del Centro de Ciencias de la Salud de la Universidad Autónoma de Aguascalientes, México

** Profesora investigadora del Departamento de Enfermería del Centro de Ciencias de la Salud de la Universidad Autónoma de Aguascalientes, México.

Fecha de recibido: 18 de mayo 2018

Fecha de aceptación: 17 de septiembre 2018

Correspondencia: MCB Silvia Patricia González Flores. Departamento de Enfermería Edificio 105, Ciudad Universitaria, Universidad Autónoma de Aguascalientes, Avenida Universidad 940, código postal 20131, Aguascalientes, Ags. México, teléfono. 910-84-37 y 44920129 17, correo electrónico silviapglzf@gmail.com
} 


\section{Introducción}

Etimológicamente la palabra calidad proviene del latín qualitas o qualitatis, que significa "perfección" y "satisfacción". A nivel de salud, ambos conceptos han tomado interés desde la década de los 80 y 90, cuando el Dr. Avedis Donabedian los introdujo a las instituciones de salud, basándose en tres pilares fundamentales: estructura, procesos y resultados, los cuales constituyen la Calidad Asistencial. ${ }^{1}$ Donabedian definió la calidad asistencial como "el modelo de asistencia esperado para maximizar el nivel de bienestar del paciente, una vez tenido en cuenta el balance de beneficios y pérdidas esperadas en todas las fases del proceso asistencial". ${ }^{2}$ La estructura la definió como las características de los escenarios donde se presta la atención y los recursos requeridos para asistencia sanitaria, el proceso se refiere a la utilización de recursos en cuanto a lo realizado en prestar y recibir asistencia; los resultados describen los efectos de la atención asistencial; los resultados describen el estado de salud de los pacientes y poblaciones, y comprende resultados definitivos ${ }^{1}$. La calidad brindada crea en el paciente una opinión sobre el cuidado recibido por parte del personal de enfermería, que no es más que su satisfacción. ${ }^{3}$ El término calidad en el campo de la salud, como en otras áreas del conocimiento, se aplica a la excelencia de una disciplina, a la perfección de un proceso, a la obtención de buenos resultados con una determinada técnica o procedimiento ${ }^{4}$. La Organización Mundial de la Salud (OMS) indica que la calidad consiste en la ejecución de acuerdo con estándares de intervenciones de probada seguridad, que son económicamente accesibles a la población en cuestión, y que poseen la capacidad de producir un impacto positivo en la mortalidad, morbilidad, discapacidad y malnutrición. ${ }^{5}$ Para González Gamboa, la calidad de servicios en salud es un producto que se brinda a usuarios individuales y colectivos de modo accesible y equitativo a través de un nivel profesional óptimo, teniendo en cuenta el balance entre beneficios, riesgos y costos, con el propósito de lograr la adhesión y satisfacción de tales usuarios. ${ }^{6}$

La enfermería es una actividad humana fundamental que se realiza desde los comienzos de la humanidad, considerada la más antigua de todas las ramas de la salud pública. La preocupación por la calidad en la asistencia sanitaria es tan antigua como el propio ejercicio médico. Los principios de la calidad en la enfermería surgen desde la propia creación de la profesión: cuidar es la esencia de la enfermería, y el cuidado es el elemento paradigmático que hace la diferencia entre enfermería y otras disciplinas del área de la salud. ${ }^{7}$

Jean Watson, en su teoría menciona que "la práctica del cuidado es central en la enfermería"; por lo tanto, enfermería como profesión dedicada al cuidado de las experiencias de salud de los seres humanos posee un rol importante dentro del equipo de salud, puesto que es quien permanece mayor tiempo con el paciente proporcionándole cuidados de enfermería que forman parte de la atención integral que se mide en las instituciones. ${ }^{8}$ 
La Organización Panamericana de la Salud menciona que el desarrollo de Programas de Garantía de calidad es una necesidad en términos de eficiencia y una obligación en términos éticos y morales, es por eso que, en México desde 2001, se desplegó en el país la Cruzada Nacional por la Calidad de los Servicios de Salud con el propósito de elevar la calidad en este ámbito. Diversas Instituciones de Salud evalúan la calidad del cuidado a través de indicadores de resultados. ${ }^{9}$ Actualmente para el proceso de la calidad, en las instituciones de salud se sustentan en los indicadores Sí Calidad, Normas Oficiales Mexicanas así como en el Programa Sectorial de Salud. Los Indicadores de Calidad ${ }^{10}$ buscan establecer mecanismos que permiten responder a las necesidades, expectativas y voz de los usuarios, este sistema se compone de nueve indicadores, dentro de los cuales el número ocho: "Atención de Enfermería en Hospitalización" se enfoca en las actividades de enfermería en un segundo nivel de atención. Una de las líneas de acción de Programa Sectorial de Salud 2013-2018 ${ }^{11}$ es asegurar el acceso efectivo a servicios de salud con calidad, mejorando la calidad de los servicios de salud del Sistema Nacional de Salud.

En México según el Instituto Nacional de Estadística y Geografía (INEGI), el porcentaje de satisfacción de los servicios de salud es del $56.8 \%$ en el Instituto de Seguridad y Servicios Sociales de los Trabajadores del Estado (ISSSTE), y de $52.7 \%$ en el Instituto Mexicano del Seguro Social (IMSS) ${ }^{12}$ y en Aguascalientes, en el segundo periodo de 2015 , el $90 \%$ de los pacientes percibió la atención recibida por parte del personal de enfermería en segundo nivel como buena a comparación de años anteriores, donde únicamente del $70 \%$ al $80 \%$ de los pacientes se encontraban satisfechos. ${ }^{13}$ Investigadores mexicanos como Blaseiro y cols $^{14}$ mencionan que el $72.74 \%$ de los pacientes consideran estar satisfechos, García Hernández y cols ${ }^{15}$ señalan que el $93.5 \%$ del personal de enfermería les informa a los pacientes lo que va a realizar antes de hacerlo.

En Colombia, $96.8 \%$ de los pacientes encuestados se encontró satisfecho con la atención de Enfermería, hallazgo que guarda relación con los altos niveles de calidad percibida en los servicios; ${ }^{16}$ Claudia Consuelo Torres y cols ${ }^{17}$ destacan la amabilidad del personal de enfermería y la privacidad proporcionada, asimismo Yeis Manuel Borré y cols ${ }^{16}$ concluyeron que la mayoría de los pacientes se encontraron satisfechos con el cuidado y la mayoría recibió información suficiente por parte del personal. J.Silva-Fhon y cols $^{18}$ concluyeron que los pacientes entrevistados tuvieron una percepción medianamente favorable respecto a la atención brindada por el profesional de enfermería, una tercera parte de los pacientes refieren que el personal no se identifica por su nombre y el $10 \%$ percibió la falta de amabilidad del personal. Dado el panorama anterior, se planteó como objetivo determinar la percepción del cuidado que tiene el paciente en relación con el servicio en el cual está hospitalizado. 


\section{Material y Métodos}

Estudio de tipo cuantitativo, transversal y correlacional, de diseño no experimental y prolectivo, realizado en un hospital público de la ciudad de Aguascalientes, con una muestra probabilística y muestreo estratificado basado en el número de camas censables en los servicios de encamados de medicina interna y encamados de cirugía $(n=42$ pacientes); cada servicio fue estrato, en el estrato del servicio de medicina interna se aplicaron 21 encuestas y en el estrato del servicio de cirugía se aplicó la misma cantidad. Se decidieron evaluar dichos servicios con el objetivo de conocer si la complejidad de los cuidados presentes en el servicio de medicina interna producía una variación en la percepción de la calidad en los cuidados de los pacientes; además, dentro de la institución fueron estos servicios los que cubrieron los criterios de selección para la aplicación del instrumento.

Se decidieron evaluar dichos servicios con el objetivo de conocer si por la complejidad de los cuidados presentes en el servicio de medicina interna, la percepción que tuviesen los pacientes de la calidad de los cuidados de enfermería podría variar, además dentro de la institución fueron estos servicios los que cubrirán los criterios de selección para la aplicación del instrumento. Los criterios de selección fueron pacientes mayores de edad, con una estancia de al menos 48 horas en el servicio, con probable alta hospitalaria y en condiciones de poder responder el cuestionario.

\section{Criterios éticos y legales}

Se consideraron los puntos veintidós, veintitrés, veinticinco y veintisiete de la declaración de Helsinki ${ }^{28}$ que mencionan que la participación de todo individuo debe ser voluntaria y debe obtenerse bajo un consentimiento informado; previo a esto, el protocolo de investigación fue evaluado tanto por la Academia de Investigación del Departamento de Enfermería de la Universidad Autónoma de Aguascalientes así como por el Comité de Investigación de la institución hospitalaria para garantizar la protección de los datos utilizados para el estudio.

Se utilizó el instrumento de medición Cuestionario de Calidad de Cuidados de Enfermería $^{19}$ (CUCACE), versión en castellano el cual fue previamente piloteado para su adaptación al lenguaje de la población mexicana, obteniendo un alfa de Cronbach de 8.7. Cuenta con dos dimensiones: la primera dimensión se titula "Experiencia en los cuidados recibidos de las enfermeras durante su estancia en la unidad"; los ítems consisten en una afirmación con siete posibilidades para responder tipo escala Likert. En total son 26 preguntas, 15 formuladas en sentido positivo y 11 en sentido negativo. Se pueden presentar para cada pregunta los resultados agrupados en tres niveles: positivo ("Un poco de acuerdo", "Muy de acuerdo "y "Completamente de acuerdo"), neutro o indiferente ("Ni de acuerdo ni en desacuerdo") y negativo ("Un poco en desacuerdo", "Muy en desacuerdo "y "Completamente en desacuerdo"). Hay que tener en cuenta que existe un número importante de preguntas que están formuladas en un sentido negativo por lo que respuestas como "Muy en desacuerdo" o "Completamente en desacuerdo" deberán ser consideradas como positivas.

La segunda dimensión es titulada "Satisfacción con los cuidados". En esta sección se pide las opiniones sobre los cuidados que ha recibido de las enfermeras durante su estancia en la unidad. Consta de 19 preguntas, con una respuesta tipo Likert de 5 opciones, todas en sentido positivo. Se pueden dividir los resultados agrupándolos en tres niveles: positivo ("Muy satisfecho "y "Completamente satisfecho"), neutro o indiferente ("Bastante satisfecho") y negativo ("Nada satisfecho "y "Poco satisfecho"); siendo confiables los instrumentos con un alfa de Cronbach de 8 y 9.4 respectivamente con un puntaje global de 
8.72. Para el análisis estadístico se utilizó el paquete Statistical Package for the Social Sciences (SPSS) v. 22 en el cual se obtuvo estadística descriptiva y estadística inferencial a través de la prueba estadística de Rho de Spearman.

\section{| | | | | | | | | | | | | | | | | | | | | | | | | | | | | | | | | | | | | | | | | | | | | | | | | | | | | | | | | | | | | | | | | | | | | | | | | | | | | | | | | | | | | | | | | | | | | | | | | | | ||}

\section{Resultados}

Se encuestaron a 42 pacientes, de los cuales el $56 \%$ correspondió al sexo femenino y el resto al sexo masculino, con una edad mínima de 18 años y una máxima de 74 años, la media de edad fue de 45.62 años, con una desviación estándar de 17.16 años. Referente a la permanencia en el servicio, contaban con 5 días de estancia en promedio, con un máximo de 23 días y un mínimo de 2, el nivel de escolaridad predominante fueron la primaria y secundaria completas con un $26.2 \%$ cada una.

Lo resultados globales mostraron que el $88.7 \%$ de los pacientes tiene experiencias positivas con el cuidado recibido por parte del personal de enfermería y el $92.2 \%$ se encuentra satisfecho con el cuidado reci- bido por parte del personal de enfermería.

El $100 \%$ de los pacientes del servicio de cirugía percibe la calidad como buena, mientras que en el servicio de medicina interna sólo el $95.2 \%$ de los pacientes percibe como buena y el $4.8 \%$ como regular. Además, en ambos servicios el $100 \%$ de los pacientes mencionó que el personal de enfermería se presentaba con ellos por su nombre.

En el apartado "Experiencia con el cuidado del personal de enfermería", se observa que en general éstas son buenas, ya que todo el personal realiza el enlace de turno frente al paciente y casi la totalidad informa correctamente al paciente antes de realizarle cualquier procedimiento ( $\mathrm{Fi}$ gura 1).

\section{Experiencia de los cuidados}

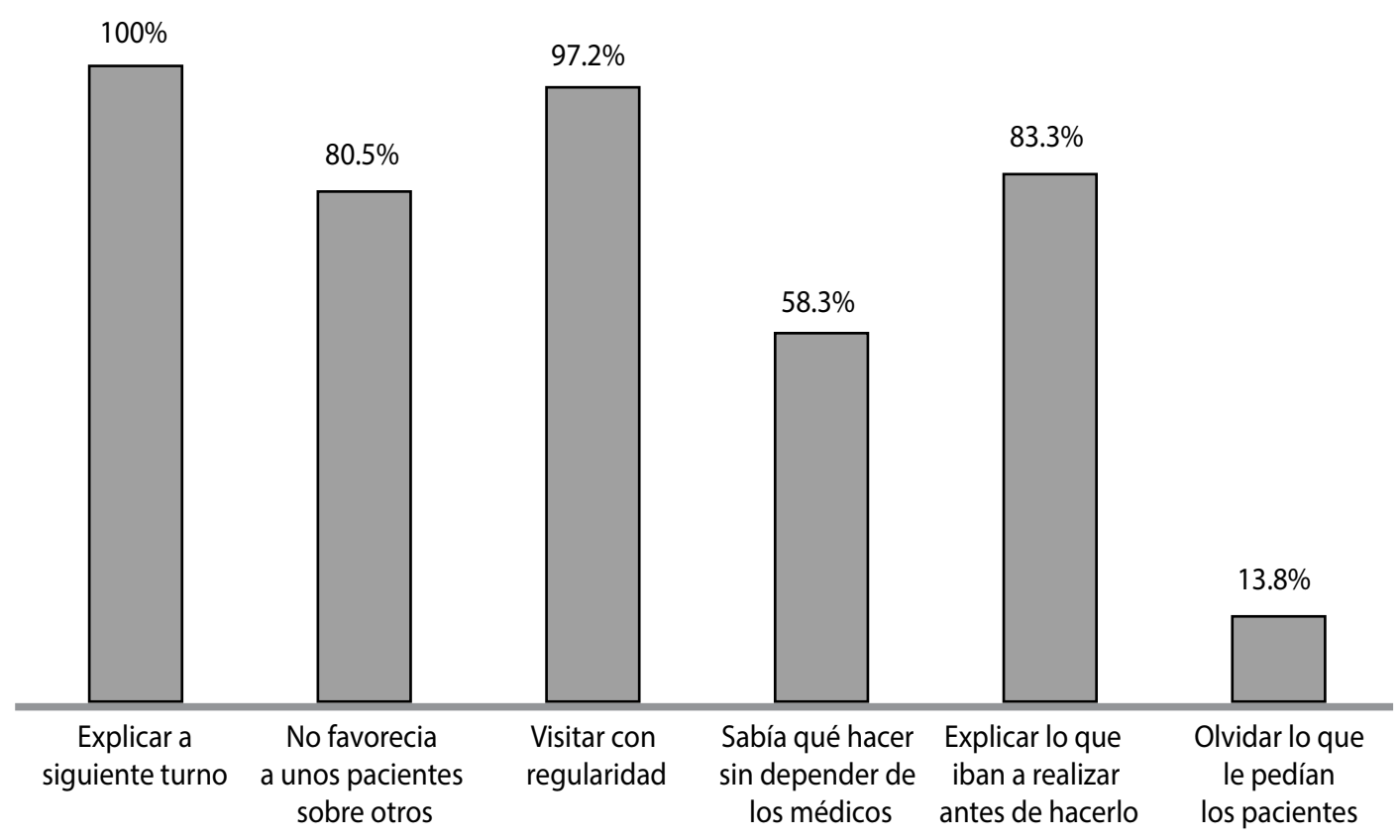

Figura 1. Puntajes obtenidos en la experiencia de los cuidados del personal de enfermería. 


\section{Grado de satisfacción}

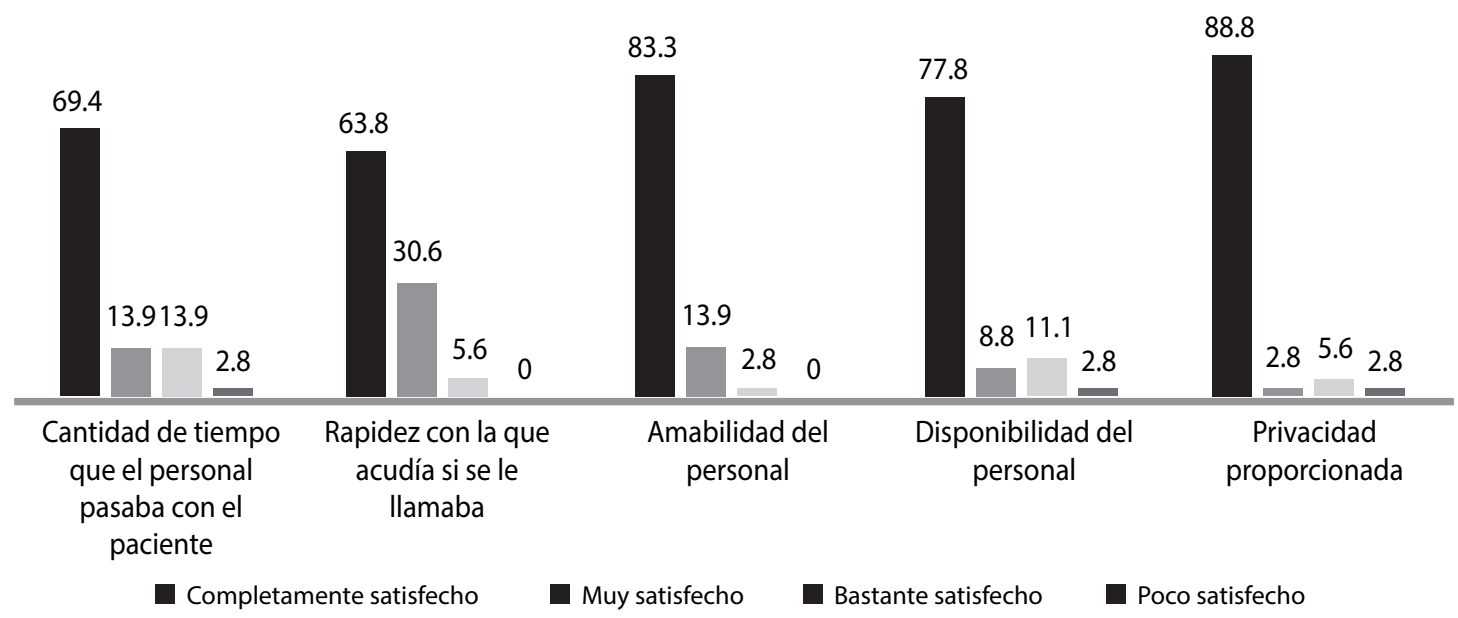

Figura 2. Grado de satisfacción con el cuidado del personal de enfermería.

Los pacientes resultaron más satisfechos con la privacidad y la amabilidad del personal y en un grado de menor satisfacción se encuentra la rapidez con la que acudían si se les llamaba o la cantidad de tiempo que el personal pasaba con ellos, esto último pudiera deberse a que el indicador enfermera - paciente es alto tomando en cuenta también el grado de complejidad o de demanda que requieren los pacientes lo que pudiera no cumplir con lo que se menciona (Figura 2).
Al realizar el contraste de hipótesis no se obtiene relación estadísticamente significativa entre las variables calidad percibida de los cuidados de enfermería por pacientes y el servicio de hospitalización, donde se obtuvo una Rho de Spearman de 0.156 con un nivel de significancia de 0.323 , por lo tanto, no existe relación positiva entre la percepción que tienen los pacientes con respecto a los cuidados de enfermería y el servicio de hospitalización en que se encuentran.

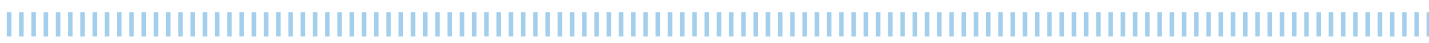

\section{Discusión}

El hablar de calidad implica retomar aspectos que influyen tanto en la estructura, proceso y resultados, mismos que pueden verse afectados por condiciones internas $y$ externas, y muchas veces ajenas al personal de enfermería y al personal de salud en general, que van más allá de la atención y se centran en suministros hospitalarios, costos de la atención, tiempo de espera entre muchos otros; sin embargo, el presente estudio se centra en la esfera de resultados del Dr. Avedis Dodabedian: como sólo se evalúa una parte de la calidad en salud, se dejan fuera otros factores que también influyen en el resultado final total de la percepción de la calidad, pero que en este estudio no eran necesarios. Debido a los criterios de inclusión necesarios para la utilización del CUCACE, otros servicios hospitalarios no fueron evaluados, lo cual limitó el estudio. El panorama de enfermería está en constante evaluación, así mismo se sigue trabajando con planes nnacionales y estratégicos para la mejora del nivel de la calidad en el cuidado en temas relacionados con la salud. 
El resultado obtenido indica que no existe relación estadística entre la calidad percibida de los cuidados de enfermería por pacientes en relación con el servicio de hospitalización; por lo tanto, el servicio en el que se encuentre hospitalizado el paciente no condiciona ni afecta el grado de calidad percibida; sin embargo, los resultados demostraron que los pacientes se encuentran satisfechos con el trato que recibieron por parte del personal de enfermería y perciben menor preparación del personal enfermero para realizar su trabajo y aún no se percibe a la enfermería como una profesión autónoma capaz de actuar sin el personal médico; se coincide con Claudia Consuelo Torres y cols ${ }^{9}$ ya que mencionan que la amabilidad del personal de enfermería y la privacidad proporcionada son de los aspectos más sobresalientes al momento de la atención, mismos ítems que se situaron dentro de los mejores puntuados, caso similar a J.Silva-Fhon y cols donde únicamente el $10 \%$ de los pacientes tuvo una percepción acerca de la fal- ta de amabilidad del personal. El profesor Yeis Manuel Borré y cols ${ }^{8}$ concluyeron que el $96.8 \%$ de los pacientes se encontró satisfecho con el cuidado recibido por parte del personal de enfermería, resultado contrario a Blaseiro y cols ${ }^{5}$ donde sólo el $72 \%$ de los pacientes se encontró satisfecho; por lo tanto, se coindice con el profesor Borré, al presentar a la mayoría de los pacientes satisfechos con el cuidado recibido por el personal de enfermería de la institución. Por otra parte, María de Lourdes García y cols, ${ }^{7}$ en un hospital de Toluca en México, al evaluar la "Calidad del cuidado de enfermería en pacientes hospitalizados con dolor", obtuvieron que el $93.5 \%$ de los enfermeros informa a los pacientes sobre lo que va a realizar antes de realizarlo, un resultado $10 \%$ arriba de lo percibido en este estudio, mientras que en el estudio realizado por Borré y cols $^{8}$ el $17 \%$ de los pacientes manifestó no haber recibido suficiente información por parte de personal de enfermería, esto en Barranquilla Colombia.

\section{Conclusiones}

Aunque algunas personas aún no desligan la profesión de enfermería con la de medicina, ni se reconoce como ciencia autónoma, los pacientes reconocen el valor que tiene la enfermería, a pesar de que pudieran existir en las instituciones de salud escasez de material, de recurso humano de salud, o condiciones que resultasen inadecuadas, el profesional de enfermería realiza su mayor esfuerzo para brindar una cuidado que garantice el bienestar propio del paciente, el cual se ve reflejado en el trato amable que percibe éste, que es casi una característica del personal de enfermería como lo muestran las investigaciones mencionadas. Además, cada día se va involucrando e incluyendo al paciente en su atención con la explicación de procedimientos que resultan o no conocidos para ellos. Actos sencillos y humanos hacen que se perciba una mejor calidad en el cuidado que brinda el personal de enfermería.

\section{Bibliografía}

1. Donabedian A, Zurita B. Calidad asistencial. Rev. Jano.1989;1: pág.35-42.

2. La calidad asistencial en la unión europea [internet].
1 ed. 2016.Disponible en: http://www.msssi.gob.es/ $\mathrm{gl} /$ organizacion/sns/informeanualsns/docs/2005/ cap3calidadasistencialeuropa.pdf

3. Cabarcas, N. Homenaje a las enfermeras: Seminario de la gestión de calidad del cuidado de enferme- 
ría. Hospital Universitario del Caribe, mayo, 2008. Disponible:www.hucaribe.gov.co/novedades/ mayo21/homenaje.pdf.

4. Lages Ruiz Julianis. Evaluación de la calidad de la atención de enfermería en el Servicio de Retina en el Instituto Cubano de Oftalmología "Ramón Pando Ferrer". Rev Cubana Oftalmol [Internet] 2016 Mar [citado 2018 abril 28];29(1): Disponible en: http://scielo.sld.cu/scielo.php?script=sci arttext\&pid=S0864-21762016000100002\&lng =es.

5. Roubier B, Luna S, Gross J, Kenton R. Evaluación de la calidad de la gestión de un laboratorio clínico hospitalario en Costa Rica. Rev Costarr Salud Pública [internet]. 2010 [citado abril 2018]; 19: 12-17 Disponible en: http://www.scielo.sa.cr/pdf/rcsp/ v19n1/a03v19n1.pdf

6. Piñeros R. Gamboa G. Calidad de atención percibida y calidad de vida relacionada con la salud en pacientes participantes de estudios clínicos de LA IPS IDEARG SAS [Doctoral]. Universidad del Rosario; 2014. citado 2018 abril 29] Disponible en http://repository.urosario.edu.co/bitstream/handle/10336/10315/1030557206-2014. pdf; sequence $=4$

7. Aranaz-Andrés JM . La calidad en los servicios sanitarios. Una propuesta general para los servicios clínicos. Rev Neurol 1999; [citado 2018 abril 28];29 (07):647-651 Disponible en: https://www.neurologia.com/articulo/99329.

8. Percepción de la calidad del cuidado de enfermería en el paciente hospitalizado. Paraninfo digital monográficos de investigación en salud [internet]. 2015 [citado 2018 abril 28] Disponible en: http://www. index-f.com/para/n22/347.php

9. Ortega MC, Suárez MG. Manual de evaluación de la calidad en enfermería. México: editorial panamericana, 2006.

10. DGCES | INDICAS II: [internet]. Dgces.salud.gob. mx. 2016.Disponible en: http://dgces.salud.gob. $\mathrm{mx}$ /indicasii/resultados.php.

11. Programa sectorial de salud 2013-2018. Consultado 28 junio 2016, disponible en http://www.conadic. salud.gob.mx/pdfs/sectorial_salud.pdf

12. Claudia Consuelo Torres Contreras.Percepción de la calidad del cuidado de enfermería en pacientes hospitalizados. Av.enferm. [Internet]. 2010 [citado 201 junio 28]; 28(2):95-107.Disponible en: https:// revistas.unal.edu.co/index.php/avenferm/article/ view/21385

13. INEGI:Instituto Nacional de Geografía y Estadística. [Internet].2016 Buscador.inegi.org.mx. Calidad de servicios de salud. Consultado: 28 abril 2016, disponible en: http://buscador.inegi.org. $\mathrm{mx} /$ search? $\mathrm{q}=\mathrm{ca}$

lidad + servicios + salud\&site $=$ sitioinegi

collection\&tx=calidad_en_salud\&client $=$ inegi_

default\&proxystylesheet $=$ inegi

default\&getfields $={ }^{*}$ \&entsp $=$ a_ inegi

politica\&lr=lang_es $\% 7$ clang_en\&filter $=1 \&$ sor

$\mathrm{t}=$ date $\% 3 \mathrm{ad} \% 3 \mathrm{al} \% 3 \mathrm{ad} 1 \& \mathrm{ie}=$ utf-8\&oe = utf-

$8 \&$ tlen $=260$

14. Balseiro Almario I, Martínez Aguilera P, Ángeles Otero R, Cortes Galicia A, De la Rosa García m, Flores Badillo A. La calidad de la atención de enfermería a los pacientes de un hospital regional del ISSSTE, en México D.F: un estudio basado en el grado de satisfacción de los usuarios. Rev Enf ENEO-UNAM [Internet]. 2007. [Consultado junio 2017]. 4(1): 9-13. Disponible en: http://dx.doi.org/10.22201/ eneo.23958421e.2007.1.30298

15. García Hernández $M$, Hernández Ortega y, Arana Gómez B, García Ortiz A. Calidad del cuidado de enfermería en pacientes hospitalizados con dolor. Horizontes en salud [internet]. 2011. [Consultado junio 2017].3(1)Disponible en: http://web.uaemex.mx/ revistahorizontes/docs/revistas/Vol3/CALIDAD_ DEL CUIDADO DE ENFERMERIA.pdf

16. Borré Ortiz Yeis Miguel, Vega Vega Yolanda. Calidad percibida de la atención de enfermería por pacientes hospitalizados. Cienc. Enferm. [internet]. 2014 dic [citado 2016 jun 28]; 20(3):81-94 Disponible en: http://dx.doi.org/10.4067/S071795532014000300008

17. Contreras C. Percepción de la calidad del cuidado de enfermería en pacientes hospitalizados. Rev UANL. [Internet].2010. Consultado junio 2016; 28(2):28110. Disponible en: http://www.revistas.unal.edu. co/index.php/avenferm/article/view/21385/22435.

18. Silva-Fhon J, Ramón-Cordova S, Vergaray-Villanueva S, Palacios-Fhon S, Partezani-Rodrigues $R$. Percepción del paciente hospitalizado respecto a la atención de enfermería en un hospital público. Enfermería universitaria. 2015;12(2):80-87. Disponible en https://doi.org/10.1016/j.reu.2015.04.001

19. Freitas Juliana Santana de, Silva Ana Elisa Bauer de Camargo, Minamisava Ruth, Bezerra Ana Lúcia Queiroz, Sousa Maiana Regina Gomes de. Quality of nursing care and satisfaction of patients attended at a teaching hospital. Rev. Latino-Am. Enfermagem [Internet]. 2014. [Consultado Julio 2016];22(3):454-460. Disponible en: http://www. scielo.br/scielo.php?script $=$ sci arttext \&pid $=$ S0104$11692014000300454 \& \operatorname{lng}=\mathrm{en}$. http://dx.doi org/10.1590/0104-1169.3241.2437. 